Article

\title{
Skp2 Regulates Subcellular Localization of PPAR $\gamma$ by MEK Signaling Pathways in Human Breast Cancer
}

\author{
Hongge Cheng ${ }^{1, \dagger}$, Jie Meng ${ }^{1, \dagger}$, Guisheng Wang ${ }^{1, \dagger}$, Yuming Meng ${ }^{1}$, Yu Li ${ }^{1}$, Dong Wei ${ }^{1}$, \\ Chunyun Fu ${ }^{1}$, Kaifeng Deng ${ }^{1}$, Aiguo Shen ${ }^{2}$, Huimin Wang ${ }^{3, *}$ and Shengming Dai ${ }^{1, *}$
}

1 Department of Laboratory Science, the Fourth Hospital Affiliated to Guangxi Medical University, Liuzhou 545005, Guangxi, China; E-Mails: ge_ge_bu_ru@sina.com (H.C.); mengjie840822@163.com (J.M.); wangguisheng@china.com (G.W.); mengyuming1@sina.com (Y.M.); 1gzhqsh@163.com (Y.L.); weidong415@126.com (D.W.); fuchunyun2008@sina.com (C.F.); lgjyk2001@163.com (K.D.)

2 Department of Immunology and Microbiology, Medical College of Nantong University, Nantong 226001, Jiangsu, China; E-Mail: shag@ntu.edu.cn

3 Medical Laboratory Center, Affiliated Hospital of Nantong University, Nantong 226001, Jiangsu, China

$\dagger$ The authors contributed equally to this work.

* Authors to whom correspondence should be addressed; E-Mails: ntfyjyk@pub.nt.jsinfo.net (H.W.); daishm@sina.com (S.D.); Tel.: +86-513-8505-2102 (H.W.); +86-772-381-5334 (S.D.); Fax: +86-513-8505-2102 (H.W.); +86-772-383-7242 (S.D.).

Received: 20 March 2013; in revised form: 15 July 2013 / Accepted: 19 July 2013 /

Published: 9 August 2013

\begin{abstract}
Nuclear hormone receptor family member PPAR $\gamma$ plays an important role in mammary gland tumorigenesis. Previous studies have shown PPAR $\gamma$ has cytoplasmic activities upon tetradecanoyl phorbol acetate (TPA) stimulation. However, the clinical pathological significance of cytoplasmic PPAR $\gamma$ is not completely understood in human breast cancer. Skp2 is oncogenic, and its frequent amplification and overexpression correlated with the grade of malignancy. In this study, the role of cytoplasmic PPAR $\gamma$ and Skp2 expression was investigated in human breast cancer progression. Therefore, immunohistochemical analysis was performed on formalin-fixed paraffin sections of 70 specimens. Furthermore, Western blot and immunofluorescence microscopy analysis were used to study the relationship between expression of cytoplasmic PPAR $\gamma$ and Skp2 expression in human breast cancer cells in vitro. Results showed that the expression
\end{abstract}


of cytoplasmic PPAR $\gamma$ was positively correlated with Skp2 expression $(p<0.05)$, and correlated significantly with estrogen receptor $(p=0.026)$ and pathological grade $(p=0.029)$, respectively. In addition, Skp2 overexpression can provoke cytoplasmic localization of PPAR $\gamma$ upon MEK1-dependent mechanisms in human breast cancer cells by nuclear-cytosolic fractionation technology and immunofluorescence microscopy analysis. Using RNA interference technology, we also found that down-regulated Skp2 reduced the phosphorylation level of MEK1 and significantly reversed TPA-induced nuclear export of PPAR $\gamma$ in MDA-MB-231 cells. The changes in the subcellular localization of PPAR $\gamma$ may represent a novel target for selective interference in patients with breast cancer.

Keywords: cytoplasmic localization; peroxisome proliferator-activated receptors $\gamma$ (PPAR $\gamma$ ); S-phase kinase protein (Skp2); human breast cancer

\section{Introduction}

Breast cancer is one of the deadliest cancers and a complex disease that results from a multi-stage process involving the deregulation of a number of different signaling cascades. Anti-estrogen hormone therapy has been best used for prevention and treatment in women with early breast cancer [1]. However, hormone therapy has little effect on estrogen receptor-negative (ER-negative) tumors [2]. Therefore, an evolving understanding of the genetic and molecular alterations in breast cancer has led to the development of novel agents that may contribute to an extension of patient survival and eventually a cure for this devastating malignancy.

Peroxisome proliferator-activated receptors (PPAR) are ligand-activated transcription factors belonging to the nuclear hormone receptor family [3,4]. Among the three PPAR isoforms (PPAR $\alpha$, $\beta / \delta$, and $\gamma$ ), PPAR $\gamma$ influences biological processes such as inflammation, cell survival, differentiation, cell proliferation and tumorigenesis [4,5]. 15-Deoxy-D12,14-prostaglandin J2(15D-PGJ2) is a natural ligand for PPAR $\gamma$ and thiazolidinediones (troglitazone as an example) are synthetic ligands for PPAR $\gamma$. PPAR $\gamma$ is highly expressed in fatty tissues and many human cancers, including breast cancer [6]. Moreover, PPAR $\gamma$ has diverse biological functions, such as promoting terminal differentiation of adipocytes, inducing differentiation and apoptosis of tumor cells and inhibiting tumor angiogenesis. These mechanisms require nuclear PPAR $\gamma$ localization, but recent data provided evidence that PPAR $\gamma$ also localized to the cytoplasm. Little is known about the mechanisms that provoke nucleocytoplasmic shuttling of PPAR $\gamma$. Recently, Burgermeister [7] reported that PPAR $\gamma$ was shuttled from the nucleus by binding its AF2 domain to MEK1, which increased the level of MEK1 phosphorylation upon TPA stimulation. This complex was actively exported from the nucleus to the cytosol by CRM1 in response to TPA stimulation, thereby causing PPAR $\gamma$ downregulation in the nucleus and inhibiting PPAR $\gamma$-dependent transactivation [8]. Khateeb et al. found that urokinase-type plasminogen activator can stimulate PPAR $\gamma$ nuclear export in hepatocytes, resulting in downregulation of paraoxonase 1 (PON1) expression [9]. However, whether cytoplasmic PPAR $\gamma$ might be important for predicting human breast cancer development and progression was not elucidated. 
S-phase kinase protein (Skp2) is oncogenic, and its frequent amplification and overexpression correlates with the grade of malignancy in certain tumors. Skp2 is the specific substrate-recognition subunit of the Skp1-Cullin-F-box protein (SCF) type ubiquitin ligase complex [10], which mediates ubiquitin-dependent degradation of some cell-cycle proteins and transcription factors, such as p27, p130, myc, and p57 [11]. Skp2 plays an important role in breast cancer, and is also considered to have strong independent prognostic potential. Signoretti et al. have recently showed that Skp2 was overexpressed in either the primary and local recurrences or metastatic breast cancers that were negative for both estrogen receptor and HER-2 receptor [12,13]. A relationship between Skp2 expression level and a poor prognosis was also observed, for example, in B-cell lymphoma [14], and breast cancer [15]. Our group has previously shown that overexpression of PPAR $\gamma$ can down-regulate Skp2 expression in MDA-MB-231 breast tumor cells [16], but whether or not Skp2 influences PPAR $\gamma$ function in breast cancer has not yet been determined.

In this study, we aim to study the relationship between expression of cytoplasmic PPAR $\gamma$ and Skp2 expression in breast cancer, and investigate the mechanisms by which Skp2 regulates cytoplasmic localization of PPAR $\gamma$. Because Skp2 is located downstream in the MAPK signaling pathway, we have in the present study tested the hypothesis that Skp2 overexpression can provoke cytoplasmic localization of PPAR $\gamma$ upon MEK1-dependent mechanisms in human breast cancer cells. Our results emphasize the importance of the cytoplasmic localization of PPAR $\gamma$ in the development and progression of breast cancer. The findings here also support a therapeutic target in this pathway for treating breast cancer.

\section{Results}

\subsection{The Expression of Cytoplasmic PPAR Is Positively Related with Skp2 Expression}

Using immunohistochemistry, we examined the clinical significance of cytoplasmic PPAR $\gamma$ and Skp2 in paraffin-embedded mammary tissue sections screened from twenty benign breast diseases and fifty breast cancer patients. We found that PPAR $\gamma$ in the benign breast disease showed positive immunoreactivity mostly in the nucleus (Figure 1A), while ER-negative breast carcinoma samples (poorly differentiated) displayed cytoplasmic mainly staining (Figure 1B). To study the pathological significance of cytoplasmic PPAR $\gamma$ in human breast cancer, the expression of PPAR $\gamma$ was independently evaluated in the cytoplasm in this study. The typical case showed that the high expression of cytoplasmic PPAR $\gamma$ was correlated with high Skp2 expression in the same breast cancer specimen (ER-negative, PR-negative, stage III) (Figure 1B,E). The expression of cytoplasmic PPAR $\gamma$ was reduced in estrogen receptor positive (ER-positive) human breast cancer (well differentiated) correlating with weak Skp2 abundance (Figure 1C,F).

To examine the relationship between the expression of cytoplasmic PPAR $\gamma$ and common parameters associated with tumor behavior, we compared cytoplasmic PPAR $\gamma$ levels with the clinicopathological features described in Table 1. A significant correlation was found among expression of cytoplasmic PPAR $\gamma$, ER expression $(p=0.026)$, and histologic grade $(p=0.029)$. Thus, the high expression of cytoplasmic PPAR $\gamma$ was associated with high histologic grade and negative ER. 
We did not observe a significant correlation among cytoplasmic PPAR $\gamma$ levels and PR status $(p=0.055)$, HER-2 status $(p=0.941), \mathrm{p} 53(p=0.164)$, TNM $(p=0.580)$, and Ki-67 $(p=0.304)$.

Table 1. The expression of cytoplasmic PPAR $\gamma$ in relation to the clinical and pathological characteristics of patients.

\begin{tabular}{|c|c|c|c|}
\hline \multirow{2}{*}{ Criteria } & \multicolumn{3}{|c|}{ Levels of cytoplasmic PPAR $\gamma$} \\
\hline & Low & High & $p$ value $^{a}$ \\
\hline \multicolumn{4}{|l|}{ ER } \\
\hline 0 & 5 & 8 & \multirow{2}{*}{$0.026 *$} \\
\hline 1 & 27 & 10 & \\
\hline \multicolumn{4}{|l|}{ PR } \\
\hline 0 & 9 & 10 & \multirow{2}{*}{0.055} \\
\hline 1 & 23 & 8 & \\
\hline \multicolumn{4}{|l|}{ HER-2 } \\
\hline Negative & 11 & 6 & \multirow{2}{*}{0.941} \\
\hline Positive & 21 & 12 & \\
\hline \multicolumn{4}{|l|}{$\mathrm{p} 53$} \\
\hline Negative & 13 & 11 & \multirow{2}{*}{0.164} \\
\hline Positive & 19 & 7 & \\
\hline \multicolumn{4}{|l|}{ TNM } \\
\hline $\mathrm{I}$ & 9 & 4 & \multirow{3}{*}{0.580} \\
\hline II & 18 & 9 & \\
\hline III & 5 & 5 & \\
\hline \multicolumn{4}{|l|}{ Grade } \\
\hline 1 & 10 & 1 & \multirow{3}{*}{$0.029 *$} \\
\hline 2 & 18 & 10 & \\
\hline 3 & 4 & 7 & \\
\hline \multicolumn{4}{|l|}{$\mathrm{Ki}-67$} \\
\hline Negative $(<19)$ & 8 & 7 & \multirow{2}{*}{0.304} \\
\hline Positive $(\geq 19)$ & 24 & 11 & \\
\hline \multicolumn{4}{|l|}{ Skp2 } \\
\hline Low $(<10)$ & 25 & 0 & \multirow{2}{*}{$0.000 *$} \\
\hline High $(\geq 10)$ & 7 & 18 & \\
\hline
\end{tabular}

${ }^{\text {a }}$ Statistical analyses were performed by the Pearson $x^{2}$ test; $* p<0.05$ is considered significant.

\subsection{Subcellular Localization of PPAR in Human Breast Cancer Cells}

To explore the significance of PPAR $\gamma$ in human breast cancer progression, we examined the subcellular distribution of endogenous PPAR $\gamma$ in human breast cancer cells. Subcellular fractionation revealed PPAR $\gamma$ localized primarily in the nucleus in MCF-7 cell lines (ER-positive), while MDA-MB-231(ER-negative) displayed mainly cytoplasmic localization (Figure 2A,B); thus, MCF-7 cell lines were used in subsequent transfection experiments. Our previous results have showed that MDA-MB-231(ER-negative) which had high invasive capacity displayed high expression levels of Skp2 [16]. Therefore, the relative abundance of cytoplasmic PPAR $\gamma$ and Skp2 appeared to exhibit a positive correlation in MDA-MB-231 cells. Next we used immunofluorescence microscopy technology 
to further confirm the results shown in Figure 2C. Indeed, we also found subcellular distribution of endogenous PPAR $\gamma$ was association with Skp2 expression.

Figure 1. Representative immunohistochemistry slides of Skp2 and PPAR $\gamma$ staining in locally advanced breast cancer. PPAR $\gamma$ and Skp2 protein expression in paraffin-embedded human breast benign and malignant tumors $(\times 400)$. (A-C) PPAR $\gamma$ reactivity; (D-F) Corresponding Skp2 staining. (A) Benign breast disease sample displaying strong nuclear staining for PPAR $\gamma$; (B) Infiltrating ductal carcinoma (ER-negative, PR-negative, and HER-2-negative, Grade III) showing strong cytoplasmic PPAR $\gamma$ immunostaining; (C) ER-positive human breast cancer sample (Grade I) showed reducing cytoplasmic PPAR $\gamma$ staining; (D) Benign breast disease sample displaying weak Skp2 immunostaining; (E) Infiltrating ductal carcinoma (ER-negative, PR-negative, and HER-2-negative, Grade III) showing strong Skp2 staining; (F) ER-positive human breast cancer sample (Grade I) showed weak Skp2 abundance.
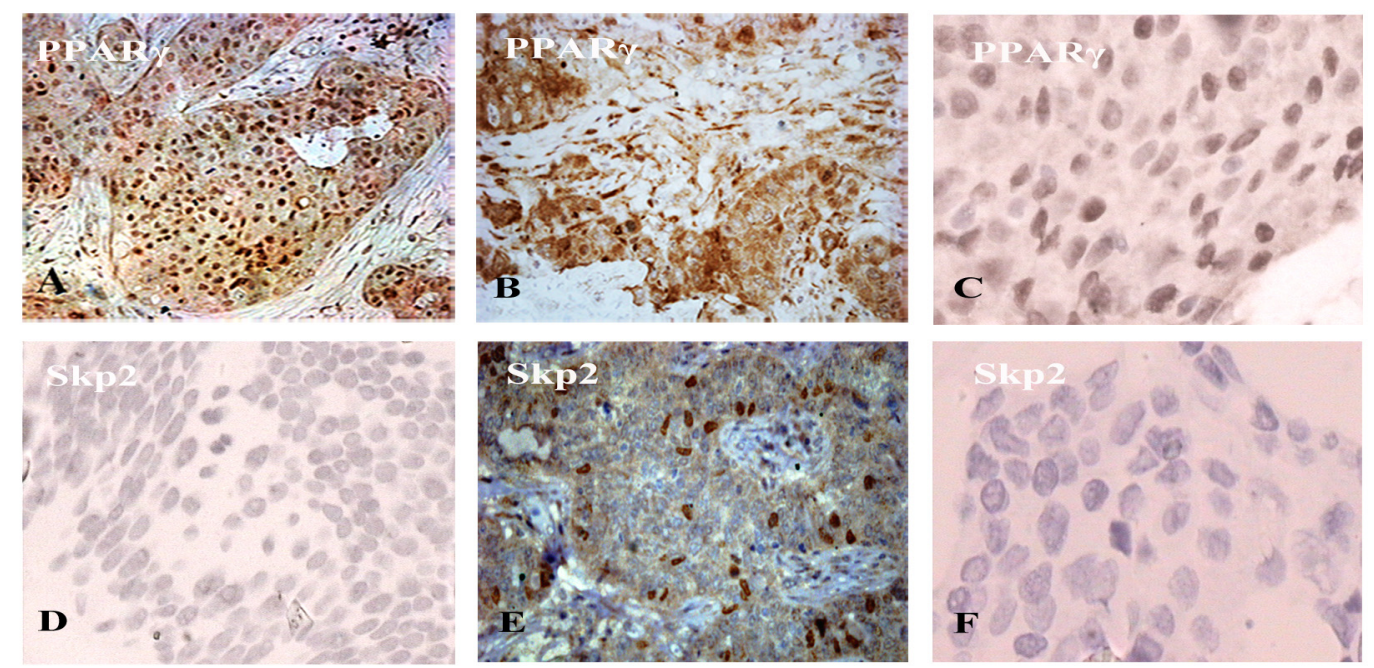

\subsection{Cytosolic Retention of PPARy upon Overexpression of Myc-Skp2}

We next sought to determine whether Skp2 can influence subcellular localization of endogenous PPAR $\gamma$. Thus, MCF-7 cells were transiently transfected with Myc-Skp2 or Myc-empty (PCDNA). Forty-eight hours after transfection, the cells were subjected to cellular fractionation. Using nuclear and cytosolic fractionation technology, a significant decrease of PPAR $\gamma$ in the nucleus was observed in Skp2-transfected cells (Figure 3A). We quantified PPAR $\gamma$ by densitometry and found that cytoplasm-localized PPAR $\gamma$ increased to about 1.6-fold in Skp2-transfected cells as compared to Myc-empty-transfected cells (Figure 3B). Moreover, immunofluorescence analysis also revealed $\operatorname{PPAR} \gamma$ was retained in the cytosol in Skp2-transfected cells (Figure 3C).

\subsection{PPAR W Was Retained in the Cytosol upon MEK1-Dependent Mechanisms in MDA-MB-231 Cells}

Extracellular signals which activate intracellular phosphorylation pathways can influence the subcellular localization of PPAR $\gamma$ [17]. Previous studies have shown that PPAR $\gamma$ can be phosphorylated by the MEK/ERK signaling pathways [7]. In this study, we found that MDA-MB-231 
cell lines displayed higher expression levels of p-MEK1 than MCF-7 cell lines. Moreover, overexpression of Myc-Skp2 resulted in increased MEK1 phosphorylation in MCF-7 cell lines (Figure 4A). Importantly, nuclear localization of PPAR $\gamma$ was induced by the MEK inhibitor PD98059 in MDA-MB-231 cells. We also found pretreatment with U0126 significantly prevented cytoplasmic localization of PPAR $\gamma$ in MDA-MB-231 cells, with a similar effect as PD98059 (Figure 4C). Then, we verified that the relocalization of PPAR $\gamma$ was mediated by MEK signaling pathways by using immunofluorescence techniques (Figure 4E). Our results showed that PPAR $\gamma$ was exported from the cytosol toward the nucleus upon stimulation with PD98059 or U0126 in MDA-MB-231 cells, indicating that MEK1 plays an important role in determining the subcellular localization of PPAR $\gamma$ in human breast cancer.

Figure 2. Subcellular localization of PPAR $\gamma$ in human breast cancer cells. (A) Equal amounts of cytosolic and nuclear lysates from MCF-7 or MDA-MB-231 cells were analyzed by Western blot to detect PPAR $\gamma$ localization. Lamin B or Tubulin were used as internal controls for nuclear or cytosolic protein, respectively; (B) Blot for PPAR $\gamma$ was quantified by densitometry and expression level relative to Lamin B or Tubulin were calculated. The data are means $\pm \operatorname{SEM}(n=3, * p<0.05$, compared with control: MCF-7); (C) MCF-7 and MDA-MB-231 cells $\left(1 \times 10^{5}\right.$ cells/well $)$ cultured in 24-well plates separately. $24 \mathrm{~h}$ later cells were fixed for detecting the subcellular location of PPAR $\gamma$ by immunofluorescence assay as described in Materials and Methods.

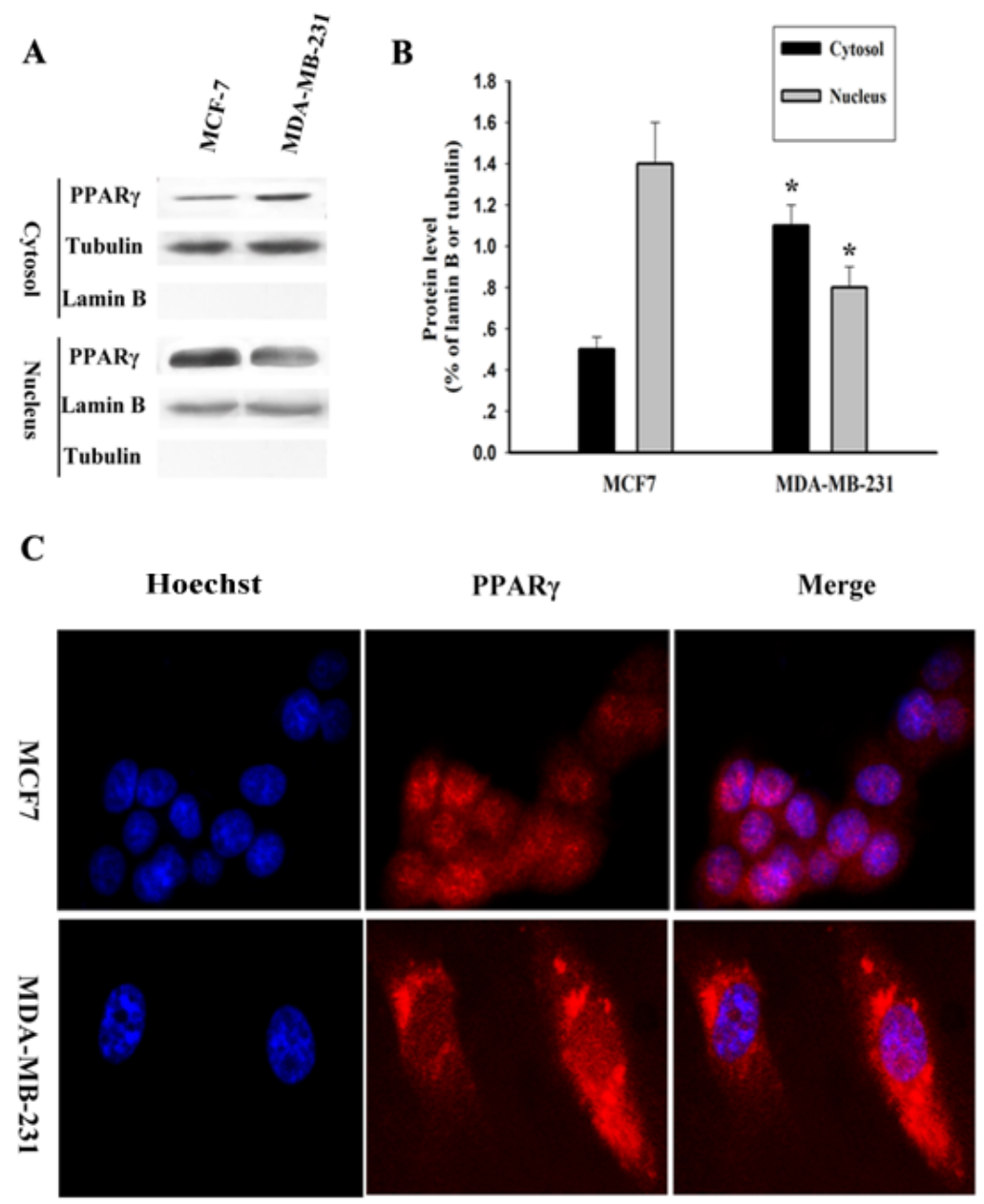


Figure 3. Cytosolic retention of PPAR $\gamma$ upon overexpression of Myc-Skp2. (A) MCF-7 cells were transiently transfected with Myc-Skp2 or Myc-empty. Forty-eight hours after transfection, the cells were subjected to cellular fractionation and analyzed by Western blot to detect PPAR $\gamma$ localization; (B) Blot for PPAR $\gamma$ was quantified by densitometry and expression level relative to Lamin B or Tubulin were calculated. The data were means $\pm \operatorname{SEM}\left(n=3,{ }^{*} p<0.05\right.$, compared with Myc-empty: PCDNA); (C) MCF-7 cells were grown on coverslips and transiently transfected with either Myc-empty or Myc-Skp2. Forty-eight hours after transfection, the cells were fixed and stained with PPAR $\gamma$ antibodies or Hoechst, and the localization was assessed by fluorescence microscopy as above.
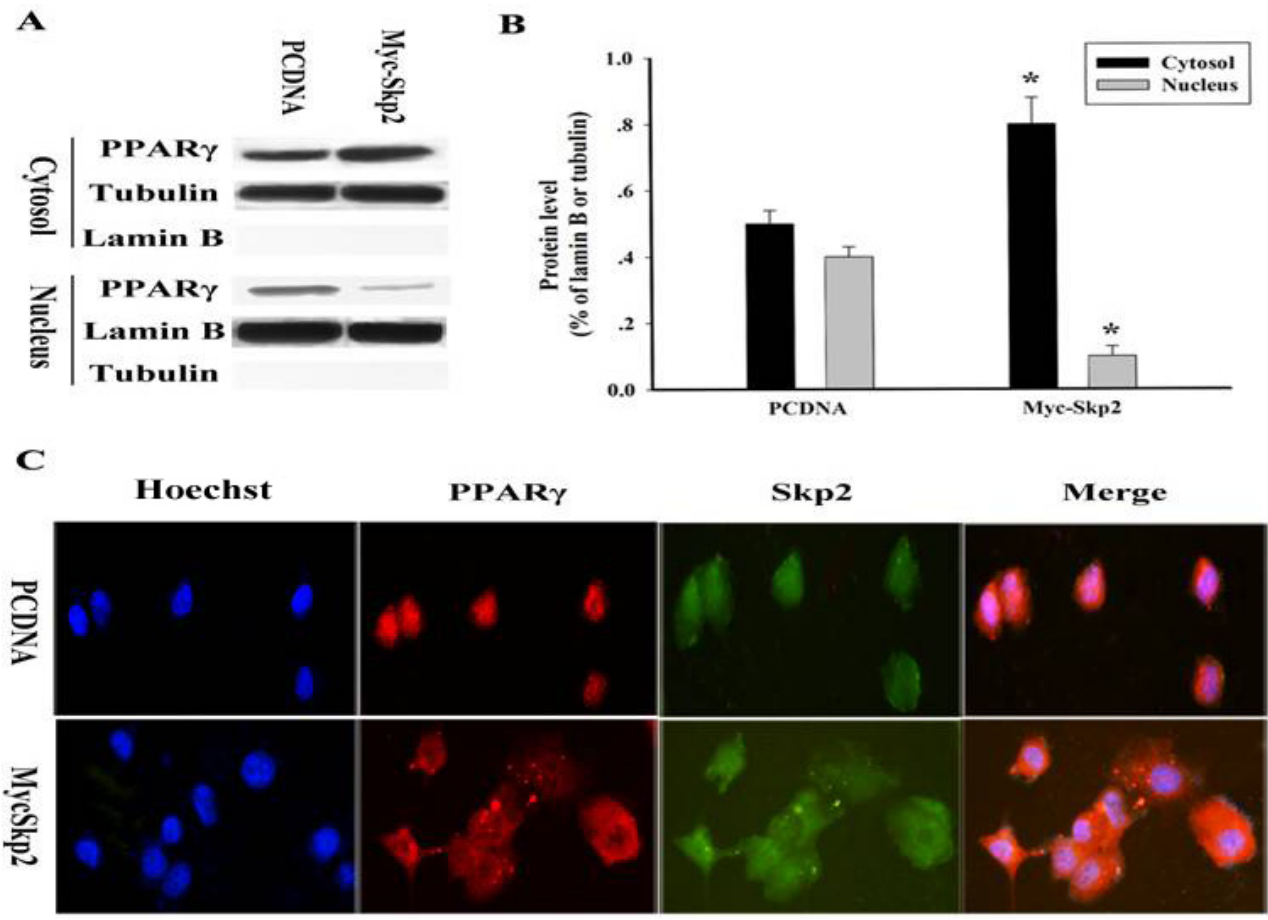

\subsection{Down-Regulated Skp2 Reduced Phosphorylation Level of MEK1 and Significantly Reversed TPA-Induced Nuclear Export of PPAR in MDA-MB-231 Cells}

To futher verify the above-mentioned results, a Skp2-shRNA expression plasmid was constructed to examine the role of Skp2 on the subcellular localization of endogenous PPAR $\gamma$. MDA-MB-231 cells were transiently transfected with Skp2-shRNA or scrambled shRNA, and the transfection efficiency was evaluated by Western blots after $48 \mathrm{~h}$ (Figure 5A). Then, we investigated the phosphorylation level of MEK1 in Skp2 shRNA-transfected cells. As shown in Figure 5B, Skp2 knockdown reduced the phosphorylation level of MEK1 in MDA-MB-231 cells compared with scrambled shRNA-transfected cells. Previous studies have shown that PPAR $\gamma$ has cytoplasmic activities upon TPA stimulation; we further investigated the relationship of TPA and Skp2-shRNA. MDA-MB-231 cells were treated with $250 \mathrm{nM}$ TPA and Skp2-shRNA alone or in combination for $30 \mathrm{~min}$. Interestingly, the combination of Skp2-shRNA with $250 \mathrm{nM}$ TPA reduced the expression levels of endogenous MEK1 by about 35\% compared with TPA-treated cells (Figure 5C). Moreover, we found that the subcellular distribution of endogenous PPAR $\gamma$ was not apparently changed in Skp2 shRNA-transfected cells compared with the scrambled shRNA-transfected cells, whereas TPA-induced 
nuclear export of PPAR $\gamma$ was reversed in Skp2 shRNA-transfected cells compared with TPA-treated cells (Figure 5D). These results suggested that down-regulated Skp2 significantly reversed TPA-induced nuclear export of PPAR $\gamma$ in MDA-MB-231 cells.

Figure 4. PPAR $\gamma$ was retained in the cytosol upon MEK1-dependent mechanisms. (A) Total proteins were extracted and MEK1/2 and their p-MEK1 detected by Western blot in MCF-7 cells, MDA-MB-231 cells, MCF-7 cells transfected with Myc-empty or Myc-Skp2; (B) Protein expression was normalized against MEK1/2. Statistical differences compared with the controls were given as * $p<0.05$; (C) MDA-MB-231 cells were treated with $10 \mu \mathrm{M}$ of MEK inhibitor PD98059 or U0126 for $12 \mathrm{~h}$, and the cells were subjected to cellular fractionation and blotted with PPAR $\gamma$ antibodies. Control cells were incubated with DMSO vehicle for the same period of time; (D) The blot for PPAR $\gamma$ was quantified by densitometry and the expression levels relative to Lamin B or Tubulin were calculated. The data are means $\pm \operatorname{SEM}(n=3, * p<0.05$, compared with control: Basal $)$; (E) MDA-MB-231 cells were treated with $10 \mu \mathrm{M}$ of MEK inhibitor PD98059 or U0126 for $12 \mathrm{~h}$, and the cells were fixed and stained with PPAR $\gamma$ antibodies or Hoechst, and the localization was assessed by fluorescence microscopy as above.

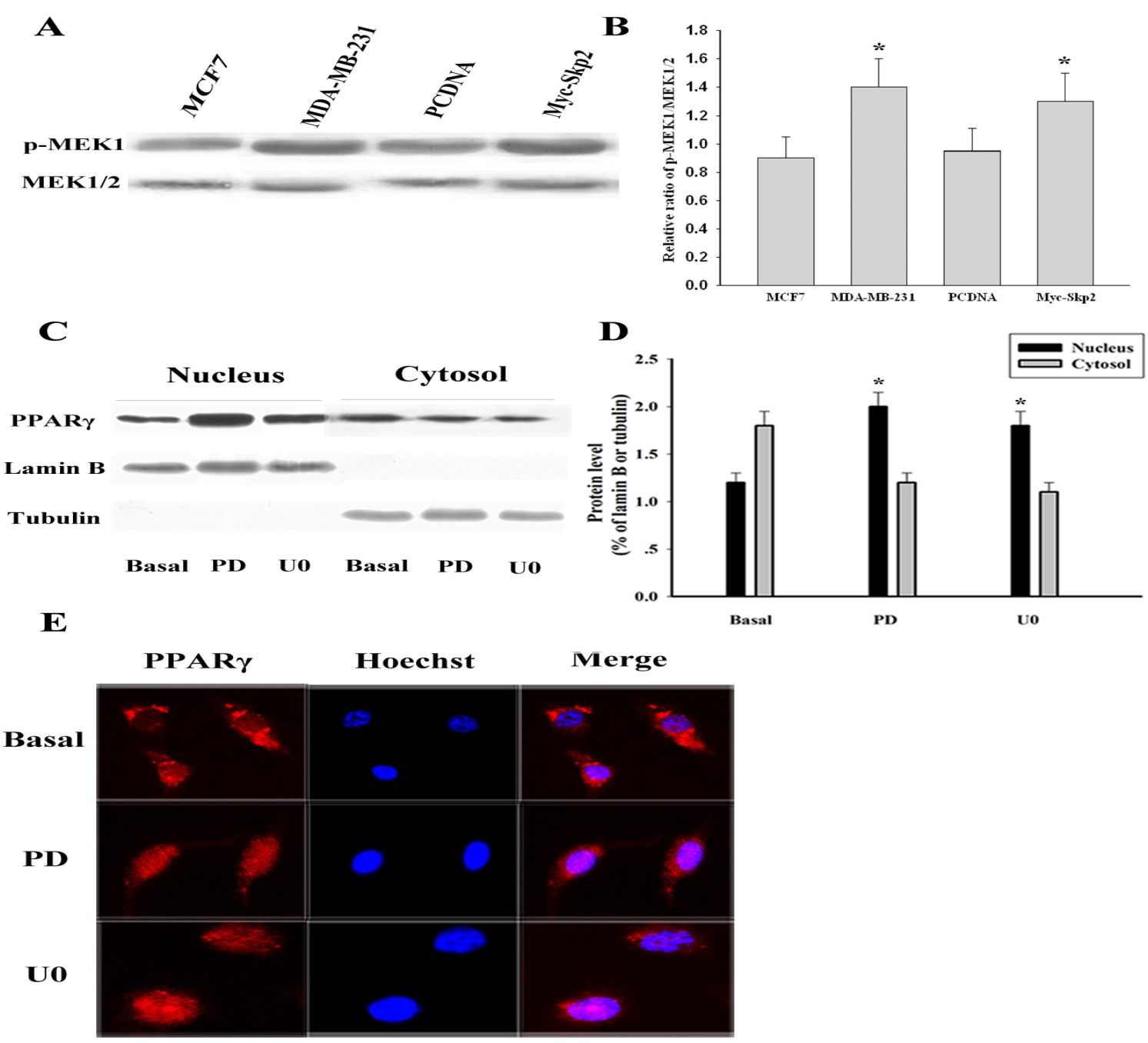


Figure 5. Down-regulated Skp2 significantly reversed TPA-induced nuclear export of PPAR $\gamma$ in MDA-MB-231 cells. (A) The cells were transfected with scrambled shRNA or Skp2 shRNA, and expression of Skp2 was evaluated by Western blot analysis; (B) MDA-MB-231 cells were transfected with either shRNA oligonucleotides of Skp2 (Skp2 shRNA) or scrambled shRNA (scramble). Forty-eight hours after transfection, the cells were serum starved $(0.1 \%$ FCS, $16 \mathrm{~h})$ and then were treated with TPA $(250 \mathrm{nM}$, $30 \mathrm{~min}$ ) or left untreated. Phosphorylation of MEK1 upon TPA stimulation of Skp2-knockdown cells was determined by pMEK and MEK $_{1 / 2}$ Abs; (C) Protein expression was normalized against MEK1/2. Statistical differences compared with the controls were given as * $p<0.05$; (D) MDA-MB-231 cells were transfected with either Skp2 shRNA or scrambled shRNA and then grown on coverslips as described above. Forty-eight hours after transfection, the cells were serum starved $(0.1 \% \mathrm{FCS}, 16 \mathrm{~h})$ and then were treated with TPA (250 nM, $30 \mathrm{~min}$ ) or left untreated. The cells were stained with polyclonal rabbit PPAR $\gamma$ and Hoechst and developed with Cy3-conjugated anti-rabbit secondary Ab. The localization of PPAR $\gamma$ was visualized by a fluorescence microscopy as above.

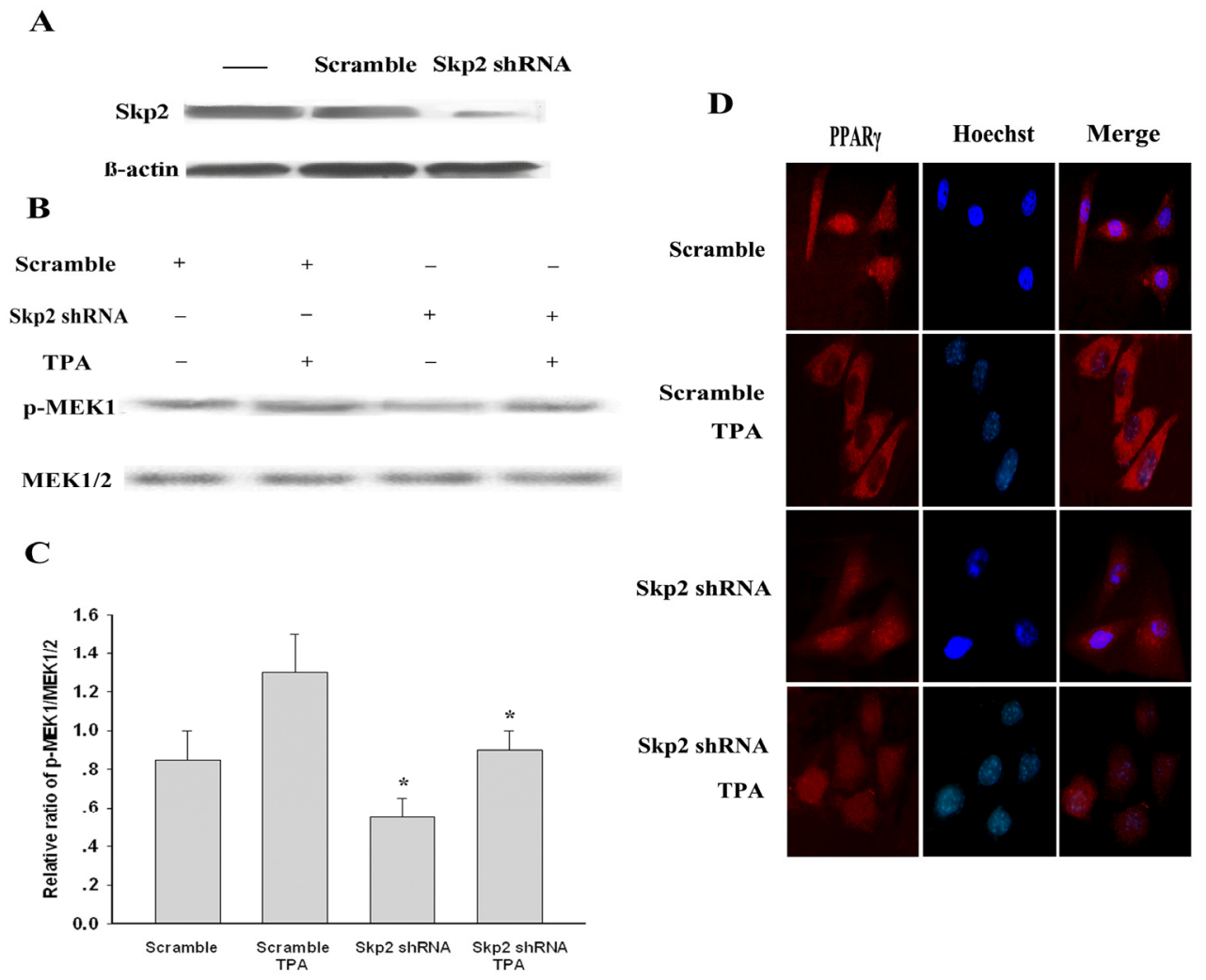

\section{Discussion}

In this report, we studied the clinical significance and relationship between cytoplasmic localization of PPAR $\gamma$ and Skp2 expression in human breast cancer. Our data revealed that expression of cytoplasmic PPAR $\gamma$ was positively related with Skp2 expression, which is critical to the development and progression of breast cancer. We demonstrated for the first time that Skp2 overexpression provokes cytoplasmic localization of PPAR $\gamma$ upon MEK1-dependent mechanisms in human breast cancer cells. 
PPAR $\gamma$ is one of the orphan nuclear receptor superfamily (NRs). It has been previously reported to reside mainly in the nucleus, similar to the vitamin D3 and thyroid hormone receptors $[18,19]$. Burgermeister et al. [7] have recently shown that PPAR $\gamma$ was exported out of the nucleus in response to TPA stimulation, and this shuttle was mediated by the nuclear export signal (NES) in the mitogen-activated protein kinase (MAPK)/extracellular signal-regulated kinase (ERK) kinase 1/2 (MEK1/2). TPA is the most commonly used phorbol ester. It can act as a tumor promoter that induces MMP-9 expression in certain cancer cells [20]. This massive nuclear export reduces the ability of PPAR $\gamma$ to transactivate nuclear target genes and thereby inhibits its genomic function [7]. In this article our results showed that MDA-MB-231(ER-negative) with high invasive capacity displayed mainly cytoplasmic localization of PPAR $\gamma$. Expression of cytoplasmic PPAR $\gamma$ was increased in poorly differentiated breast carcinoma samples, which was similar to the reports of other research groups [21].

Recently, a growing body of research data has shown that Skp2 plays an oncogenic role and its expression may contribute to the development and progression of human cancers [22]. Overexpression of Skp2 has been reported in several human malignant tumors, including oral squamous cell carcinoma [14,23], ovarian adenocarcinoma [24], lymphoma [25], colorectal carcinomas [26,27], gastric carcinoma [28], soft tissue sarcomas [29], acute myelogenous leukemia [30], and breast cancer [31]. In this study, we found that the expression of cytoplasmic PPAR $\gamma$ was positively related with Skp2 expression $(p<0.05)$ and Skp2 overexpression can provoke nucleocytoplasmic shuttling of PPAR $\gamma$. This suggests that Skp2 up-regulation plays an important role in reducing the ability of PPAR $\gamma$ to transactivate nuclear target genes. In other words, cytoplasmic PPAR $\gamma$ may lose the ability to reduce cell proliferation and induction of apoptosis in breast cancer cells. However, more studies are required to fully understand the roles of cytoplasmic PPAR $\gamma$ in human breast cancer.

Multiple studies have shown that ERK activities were up-regulated in many human cancers including breast cancer, and elevated ERK activity in human tumors has been correlated with poor prognosis, demonstrating that ERK may play a crucial role in human tumorigenesis [32]. The fact that Skp2 expression was positive correlated with phospho-MAPK/ERK1/2 expression during progression of cervical neoplasia was in accordance with the study of human breast cancer progression [33]. Our results showed that MDA-MB-231 cell lines displayed higher expression level of p-MEK1 than MCF-7 cell lines. Moreover, overexpression Myc-Skp2 resulted in increased MEK1 phosphorylation in MCF-7 cell lines. Importantly, nucleic localization of PPAR $\gamma$ was induced by the MEK inhibitor, (PD98059, U0126), in MDA-MB-231cells. Using RNA interference technology, we found that down-regulated Skp2 reduced the phosphorylation level of MEK1 and significantly reversed TPA-induced nuclear export of PPAR $\gamma$ in MDA-MB-231 cells. It is also possible that the regulation of PPAR $\gamma$ subcellular localization was accomplished by other mechanisms; the mechanisms should be further investigated. These findings suggest that PPAR $\gamma$ is retained in the cytosol upon MEK1-dependent mechanisms in MDA-MB-231 cells. 


\section{Experimental Section}

\subsection{Materials}

The antibodies used for immunohistochemistry in this study included: anti-PPAR $\gamma$ (sc-7196, 1:100, Santa Cruz, Biotechnology, Dallas, TX, USA), and anti-Skp2 (sc-74477, 1:100, Santa Cruz, Biotechnology, Dallas, TX, USA). Antibodies for Western blot included: anti-Skp2 (sc-7164, 1:500, Santa Cruz, Biotechnology, Dallas, TX, USA), anti-PPAR $\gamma$ (sc-7196, 1:500, Santa Cruz, Biotechnology, Dallas, TX, USA), anti-pMEK1 (sc-271914, 1:1000, Santa Cruz, Biotechnology, Dallas, TX, USA), anti-MEK1/2 (sc-436, 1:250, Santa Cruz, Biotechnology, Dallas, TX, USA), anti-Lamin B and anti-Tubulin were purchased from Santa Cruz Biotechnology (CA), PD98059, U0126 and tetradecanoyl phorbol acetate (TPA) were from Sigma (St. Louis, MO, USA), B-actin (sc-7196, 1:1000, Santa Cruz, Biotechnology, Dallas, TX, USA).

\subsection{Cell Culture}

Two human breast cancer cell lines: MCF-7(ER-positive), MDA-MB-231(ER-negative), which were gifts from the Department of Oncology, Cancer Hospital of Fudan University were used in this study. All cell lines were maintained in RPMI 1640 (Gibco BRL, Grand Island, NY, USA) supplemented with $10 \%$ heat-inactivated fetal calf serum, $2 \mathrm{mM}$ L-glutamine, and $100 \mathrm{U} / \mathrm{mL}$ penicillin-streptomycin mixture (Gibco BRL, Grand Island, NY, USA) at $37{ }^{\circ} \mathrm{C}$ and $5 \% \mathrm{CO}_{2}$.

\subsection{Tissue Samples}

Fifty breast cancer and twenty benign breast disease specimens from patients who underwent surgery between 2005 and 2008 at the Department of General Surgery, Affliated Hospital of Nantong University were formalin-fixed and paraffin-embedded for histopathologic diagnosis and immunohistochemical study. Fresh samples were frozen in liquid nitrogen immediately after surgical removal and maintained at $-80{ }^{\circ} \mathrm{C}$ until use for Western blot analysis. All human tissue was collected using protocols approved by the Ethics Committee of Affiliated Cancer Hospital of Nantong University.

\subsection{Immunohistochemistry Methods}

Serial sections that were $4 \mu \mathrm{m}$ thick were mounted on glass slides coated with $10 \%$ polylysine. Sections were dewaxed in xylene and rehydrated in graded ethanols. Endogenous peroxidase activity was blocked by immersion in $0.3 \%$ methanolic peroxide for $40 \mathrm{~min}$. Immunoreactivity was enhanced by microwaving by incubating the tissue sections for $10 \mathrm{~min}$ in $0.1 \mathrm{M}$ citrate buffer. Immunostaining was performed using the avidin biotin peroxidase complex method and antigen-antibody reactions were visualized with the chromogen diaminobenzadine.

\subsection{Immunohistochemical Evaluation}

Scoring of immunohistochemical slides was done according to the percentage of tumor cells exhibiting nuclear staining. Two independent pathologists (SP and FM) evaluated the immunostaining. At least ten high-power fields were randomly chosen and at least 300 cells/field was counted in each 
section. PPAR $\gamma$ positivity was defined as immunoreactive in $>5 \%$ and negative in no staining or $<5 \%$ of the cancer cells [21]. A cut-off value of $10 \%$ in 10 high-power fields was used to define Skp2 staining [12,34,35]. Immunohistochemical evaluation for ER, PR, p53, Ki-67 and HER-2 were finished by Department of Pathology, Affiliated Hospital of Nantong University.

\subsection{Expression Plasmid and Transient Transfection}

The full-length human Skp2 complementary DNA (cDNA) was amplified by PCR using two oligonucleotides (5'-GCGAATTCATGCACGTATTTTAAACTCC-3') and (5'-GACTCGAGACTTCATAGACAACTGGGCT-3') and cloned into pcDNA3.1-myc expression vector at the EcoR I/Xho I. Transfection was performed using lipofectamine 2000 transfection reagent (Invitrogen, Grand Island, NY, USA) according to the manufacture's protocol with minor modifications. MCF-7 cells were seeded at $2 \times 10^{5}$ cells $/ \mathrm{mL}$ in a six-well plate $24 \mathrm{~h}$ before transfection. $500 \mu \mathrm{L}$ of transfection mixture containing $8 \mu \mathrm{g}$ of DNA and $20 \mu \mathrm{L}$ of lipofectamine 2000 reagent in $450 \mu \mathrm{L}$ of Opti-MEM (Invitrogen, Grand Island, NY, USA) were added to each well. The cells were harvested $48 \mathrm{~h}$ after transfection and used for the experiment. The experiments were repeated at least three times.

\subsection{Cellular Fractionation}

Cells were grown in $10 \mathrm{~cm}$ plates to subconfluence. Subsequently, the cells were washed with PBS and scraped into ice-cold buffer $\mathrm{H}$ (50 mM-glycerophosphate, $\mathrm{pH}$ 7.3, $1.5 \mathrm{mM}$ EGTA, $1 \mathrm{mM}$ EDTA, $1 \mathrm{mM}$ dithiothreitol, $0.1 \mathrm{mM}$ sodium vanadate, $1 \mathrm{mM}$ benzamidine, $10 \mu \mathrm{g} / \mathrm{mL}$ aprotinin, $10 \mu \mathrm{g} / \mathrm{mL}$ leupeptin, and $2 \mu \mathrm{g} / \mathrm{mL}$ pepstatin A). The cells were then spun down $(12,000 \times \mathrm{g}, 5 \mathrm{~min})$, resuspended in $0.1 \%$ NP-40, and spun down again. The supernatant containing the cytosolic fraction, was boiled in sample buffer. The pellet containing the nuclei, was resuspended in an extraction buffer $(420 \mathrm{mM}$ $\mathrm{NaCl}$, 50 mM-glycerophosphate, $0.5 \mathrm{mM} \mathrm{Na} \mathrm{VO}_{4}, 1.5 \mathrm{mM} \mathrm{MgCl}, 0.2 \mathrm{mM}$ EDTA, $1 \mathrm{mM}$ dithiothreitol, 25\% glycerol), and disrupted by sonication (twice for $10 \mathrm{~s}$ ). The extract was then cleared by centrifugation $(12,000 \times \mathrm{g}, 5 \mathrm{~min})$, and the supernatant was boiled in sample buffer as the nuclear fraction. Equal amounts of protein of nucleus and cytoplasm were then separated on $10 \%$ SDS-polyacrylamide gel electrophoresis.

\section{8. shRNAs and Transfection}

The human Skp2 shRNA expression vector, pGenesil 1.2-EGFP, was constructed. This shRNA targeting the nucleotide residues 5'-AAGGAGATGTCCATGTCCAAG-3' and 5'-GCCTAAGCTAAATCGAGAGAA-3' was constructed. MDA-MB-231 cells were seeded the day before transfection using 1640 with 10\% FBS without antibiotics. Transient transfection of shRNA vectors and scrambled shRNA vectors were carried out using lipofectamine 2000 and plus reagent in Opti-MEM as suggested by the manufacturer. Cells were incubated with the pGenesil 1.2 vectors and lipofectamine and plus reagent complexes for $4 \mathrm{~h}$ at $37^{\circ} \mathrm{C}$. FBS was then added to the cells to achieve a final concentration of $10 \%$ in DMEM. Transfected cells were used for subsequent experiments $48 \mathrm{~h}$ after transfection. 


\subsection{Western Blot Analysis}

Prior to immunoblotting, cells were washed three times with ice-cold PBS, resuspended in $2 \times 1$ lysis buffer (50 mM Tris- $\mathrm{HCl}, 120 \mathrm{mM} \mathrm{NaCl}, 0.5 \%$ Nonidet P-40, $100 \mathrm{mM} \mathrm{NaF}, 200 \mu \mathrm{M} \mathrm{Na}_{3} \mathrm{VO}_{4}$, and protease inhibitor mixture) or frozen tissues were homogenized in lysis buffer (1\% NP-40, $50 \mathrm{mmol} / \mathrm{L}$ Tris, $\mathrm{pH} 7.5,5 \mathrm{mmol} / \mathrm{L}$ EDTA, 1\% SDS, 1\% sodium deoxycholate, $1 \%$ Triton X-100, $1 \mathrm{mmol} / \mathrm{L}$ PMSF, $10 \mathrm{mg} / \mathrm{mL}$ aprotinin, and $1 \mathrm{mg} / \mathrm{mL}$ leupeptin) and then incubated for $20 \mathrm{~min}$ at $4{ }^{\circ} \mathrm{C}$ while rocking. Lysates were cleared by centrifugation $\left(10 \mathrm{~min} \times 12,000 \mathrm{rpm}, 4{ }^{\circ} \mathrm{C}\right)$. Western blot procedure was performed as described previously [36]. $50 \mu \mathrm{g}$ of total protein was resolved by SDS-PAGE and transferred onto polyvinylidene difluoride membranes (Immobilon, Millipore). The membranes were first blocked with 5\% nonfat dry milk and then incubated with the primary antibody described above for $2 \mathrm{~h}$ at room temperature. After three washes, filters were incubated with horseradish peroxidase-conjugated secondary human anti-mouse or anti-rabbit antibodies (1:1000; pierce) for $1 \mathrm{~h}$ at room temperature according to the manufacturer's instructions. Detection of immunocomplexes was performed with an enhanced chemiluminescence system (NEN Life Science Products, Boston, MA, USA).

\subsection{Immunofluorescence Microscopy}

Cells were fixed on coverslips in 3\% paraformaldehyde in PBS, followed by a $10 \mathrm{~min}$ permeabilization in $1 \%$ Triton X-100 in PBS at $23{ }^{\circ} \mathrm{C}$. Then all cells were blocked with $10 \%$ normal serum blocking solution-species the same as the secondary antibody, containing $3 \%$ bovine serum albumin (BSA) and $0.1 \%$ Triton X-100 and 0.05\% Tween-20 two hours at room temperature in order to avoid unspecific staining. Then the coverslips were incubated overnight at $4{ }^{\circ} \mathrm{C}$ with primary antibodies against PPAR $\gamma$. On the following day, Cy3 conjugated (1:1000, Jackson lab) secondary antibodies were added in dark room and incubated for $2-3 \mathrm{~h}$ at $4{ }^{\circ} \mathrm{C}$. After three washes in PBS, the cells were mounted onto slides with glycerol containing 1, 4-diazobicyclo $(2,2,2)$ octane (DABCO), which were observed under a Leica fluorescence microscope (Leica Microsystems, Wetzlar, Germany).

\subsection{Statistical Analysis}

All computations were carried out using the state 7.0 statistical program. Data were presented as mean \pm standard error (se) values of $n$ independent determinations and were triplicated within each experiment. Comparisons were analyzed by using one-way analysis of variance followed by the posteriori Student-Newman-Keul $t$-test. A value of $p<0.05$ was considered significantly.

\section{Conclusions}

Generally speaking, cytoplasmic localization of PPAR $\gamma$ could become an important therapeutic target in breast cancer for several reasons. First, the expression of cytoplasmic PPAR $\gamma$ was positively related with Skp2 expression $(p<0.05)$ and correlated significantly with estrogen receptor $(p=0.026)$, pathological grade $(p=0.029)$. However, hormone therapy has little effect on ER-negative tumors. Second, Skp2 induced an export of PPAR $\gamma$ as observed above, and this reduced the amount of active PPAR $\gamma$ in the nucleus and thereby inhibited its genomic function. Third, MDA-MB-231(ER-negative) displayed mainly cytoplasmic localization of PPAR $\gamma$. Finally, the MEK-ERK signaling pathway has 
been shown to play a critical role in the survival and growth of breast cancer cells. Thus, it may be a new idea for the ER-negative breast cancer prevention and treatment to induce PPAR $\gamma$ nuclear export. Next, we are planning to study the correlation between cytoplasmic PPAR $\gamma$ expression and patient survival further, and develop a MEK-ERK pathway signature in breast cancer cells that could be used for patient selection for treatment with a MEK inhibitor.

\section{Acknowledgments}

This work was supported by grants from National Natural Science Foundation of China (No.81260395); Natural Science Foundation of Guangxi Zhuang Autonomous Region (2012jjAA40623); Health Department of Jiangsu Province (H200760) and Key Subject of Jiangsu Province (xk200723).

\section{Conflict of Interest}

The authors declare no conflict of interest.

\section{References}

1. Houssami, N.; Cuzick, J.; Dixon, J.M. The prevention, detection, and management of breast cancer. Med. J. Aust. 2006, 184, 230-234.

2. Cuzick, J.; Warwick, J.; Pinney, E.; Warren, R.M.; Duffy, S.W. Tamoxifen and breast density in women at increased risk of breast cancer. J. Natl. Cancer Inst. 2004, 96, 621-628.

3. Mangelsdorf, D.J.; Thummel, C.; Beato, M.; Herrlich, P.; Schütz, G.; Umesono, K.; Blumberg, B.; Kastner, P.; Mark, M.; Chambon, P.; et al. The nuclear receptor superfamily: The second decade. Cell 1995, 83, 835-839.

4. Kliewer, S.A.; Forman, B.M.; Blumberg, B.; Ong, E.S.; Borgmeyer, U.; Mangelsdorf, D.J.; Umesono, K.; Evans, R.M. Differential expression and activation of a family of murine peroxisome proliferator-activated receptors. Proc. Natl. Acad. Sci. USA 1994, 91, 7355-7359.

5. Panigrahy, D.; Huang, S.; Kieran, M.W.; Kaipainen, A. PPAR $\gamma$ as a therapeutic target for tumor angiogenesis and metastasis. Cancer Biol. Ther. 2005, 4, 687-693.

6. Ikezoe, T.; Miller, C.W.; Kawano, S.; Heaney, A.; Williamson, E.A.; Hisatake, J.; Green, E.; Hofmann, W.; Taquchi, H.; Koeffler, H.P. Mutational analysis of the peroxisome proliferator-activated receptor $\gamma$ gene in human malignancies. Cancer Res. 2001, 61, 5307-5310.

7. Burgermeister, E.; Chuderland, D.; Hanoch, T.; Meyer, M.; Liscovitch, M.; Seger, R. Interaction with MEK causes nuclear export and downregulation of peroxisome proliferator-activated receptor $\gamma$. Mol. Cell. Biol. 2007, 27, 803-817.

8. Knethen, A.V.; Tzieply, N.; Jennewein, C.; Brüne, B. Casein-kinase-II-dependent phosphorylation of PPAR $\gamma$ provokes CRM1-mediated shuttling of PPAR $\gamma$ from the nucleus to the cytosol. J. Cell. Sci. 2010, 123, 192-201.

9. Khateeb, J.; Kiyan, Y.; Aviram, M.; Tkachuk, S.; Dumler, I.; Fuhrman, B. Urokinase-type plasminogen activator downregulates paraoxonase 1 expression in hepatocytes by stimulating peroxisome proliferator-activated receptor- $\gamma$ nuclear export. Arterioscler. Thromb. Vasc. Biol. 2012, 32, 449-458. 
10. Krek, W. Proteolysis and the G1-S transition: the SCF connection. Curr. Opin. Genet. 1998, 8, $36-42$.

11. Nakayama, K.I.; Nakayama, K. Ubiquitin ligases: Cell-cycle control and cancer. Nat. Rev. Cancer 2006, 6, 369-381.

12. Signoretti, S.; Di Marcotullio, L.; Richardson, A.; Richardson, A.; Isaac, B.; Rue, M.; Monti, F.; Loda, M.; Pagano, M. Oncogenic role of the ubiquitin ligase subunit Skp2 in human breast cancer. J. Clin. Invest. 2002, 110, 633-641.

13. Gstaiger, M.; Jordan, R.; Lim, M.; Catzavelos, C.; Mestan, J.; Slingerland, J.; Krek, W. Skp2 is oncogenic and overexpressed in human cancers. Proc. Natl. Acad. Sci. USA 2001, 98, 5043-5048.

14. Seki, R.; Ohshima, K.; Fujisaki, T.; Uike, N.; Kawano, F.; Gondo, H.; Makino, S.; Eto, T.; Moriuchi, Y.; Taguchi, F.; et al. Prognostic significance of S-phase kinase-associated protein 2 and p27kip1 in patients with diffuse large B-cell lymphoma: effects of rituximab. Ann. Oncol. 2010, 21, 833-841.

15. Traub, F.; Mengel, M.; Lück, H.J.; Kreipe, H.H.; von Wasielewski, R. Prognostic impact of Skp2 and p27 in human breast cancer. Breast Cancer Res. Treat. 2006, 99, 185-191.

16. Meng, J.; Ding, Y.; Shen, A.; Yan, M.; He, F.; Ji, H.; Zou, L.; Liu, Y.; Wang, Y.; Lu, X.; et al. Overexpression of PPAR $\gamma$ can down-regulate Skp2 expression in MDA-MB-231 breast tumor cells. Mol. Cell Biochm. 2010, 345, 171-180.

17. Burns, K.A.; Vanden Heuvel, J.P. Modulation of PPAR activity via phosphorylation. Biochim. Biophys. Acta 2007, 1771, 952-960.

18. Berger, J.; Patel, H.V.; Woods, J.; Hayes, N.S.; Parent, S.A.; Clemas, J.; Leibowitz, M.D.; Elbrecht, A.; Rachubinski, R.A.; Capone, J.P.; et al. A PPARgamma mutant serves as a dominant negative inhibitor of PPAR signaling and is localized in the nucleus. Mol. Cell. Endocrinol. 2000, $162,57-67$.

19. Gurnell, M.; Wentworth, J.M.; Agostini, M.; Adams, M.; Collingwood, T.N.; Provenzano, C.; Browne, P.O.; Rajanayagam, O.; Burris, T.P.; Schwabe, J.W. A dominant-negative peroxisome proliferator-activated receptor gamma (PPARgamma) mutant is a constitutive repressor and inhibits PPARgamma-mediated adipogenesis. J. Biol. Chem. 2000, 275, 5754-5759.

20. Kim, S.; Choi, J.H.; Lim, H.I.; Lee, S.K.; Kim, W.W.; Kim, J.S.; Kim, J.H.; Choe, J.H.; Yang, J.H. The Raf/MEK/ERK pathway in MCF-7 human breast cancer cells. Phytomedicine 2009, 16, 573-580.

21. Papadaki, I.; Mylona, E.; Giannopoulou, I.; Markaki, S.; Keramopoulos, A.; Nakopoulou, L. PPARgamma expression in breast cancer: clinical value and correlation with ERbeta. Histopathology 2005, 46, 37-42.

22. Mani, A.; Gelmann, E.P. The ubiquitin-proteasome pathway and its role in cancer. J. Clin. Oncol. 2005, 23, 4776-4789.

23. Kudo, Y.; Kitajima, S.; Sato, S.; Miyauchi, M.; Ogawa, I.; Takata, T. High expression of S-phase kinase-interacting protein 2, human F-box protein, correlate with poor prognosis in oral squamous cell carcinomas. Cancer Res. 2001, 61, 7044-7047.

24. Malik, S.N.; Brattain, M.; Ghosh, P.M.; Troyer, D.A.; Prihoda, T.; Bedolla, R.; Kreisberg, J.I. Immunohistochemical demonstration of phospho-Akt in high Gleason grade prostate cancer. Clin. Cancer Res. 2002, 8, 1168-1171. 
25. Latres, E.; Chiarle, R.; Schulman, B.A.; Pavletich, N.P.; Pellicer, A.; Inghirami, G.; Pagano, M. Role of the F-box protein Skp2 in lymphomagenesis. Proc. Natl. Acad. Sci. USA 2001, 98, 2515-2520.

26. Hershko, D.; Bornstein, G.; Ben-Izhak, O.; Carrano, A.; Pagano, M.; Krausz, M.M.; Hershko, A. Inverse relation between levels of p27 (Kip1) and of its ubiquitin ligase subunit Skp2 in colorectal carcinomas. Cancer 2001, 91, 1745-1751.

27. Shapira, M.; Ben-Izhak, O.; Linn, S.; Futerman, B.; Minkov, I.; Hershko, D.D. The prognostic impact of the ubiquitin ligase subunit Skp2 and Cks1 in colorectal carcinoma. Cancer 2005, 103, 1336-1346.

28. Masuda, T.; Inoue, H.; Sonoda, H.; Mine, S.; Yoshikawa, Y.; Nakayama, K.; Nakayama, K.; Mori, M. Clinical and biological significance of S-phase kinase-associated protein 2 (Skp2) gene expression in gastric carcinoma: Modulation of malignant phenotype by Skp2 overexpression, possibly via p27 proteolysis. Cancer Res. 2002, 62, 3819-3825.

29. Oliveira, A.M.; Okuno, S.H.; Nascimento, A.G. Lloyd RVSkp2 protein expression in soft tissue sarcomas. J. Clin. Oncol. 2003, 21, 722-727.

30. Min, Y.H.; Cheong, J.W.; Lee, M.H.; Kim, J.Y.; Lee, S.T.; Hahn, J.S.; Ko, Y.W. Elevated S-phase kinase-associated protein 2 protein expression in acute myelogenous leukemia: Its association with constitutive phosphorylation of phosphatase and tensin homologue protein and poor prognosis. Clin. Cancer Res. 2004, 10, 5123-5130.

31. Radke, S.; Pirkmaier, A.; Germain, D. Differential expression of the F-box proteins Skp2 and Skp2B in breast cancer. Oncogene 2005, 24, 3448-3458.

32. Kohno, M.; Pouyssegur, J. Targeting the ERK signaling pathway in cancer therapy. Ann. Med. 2006, 38, 200-211.

33. Adeyinka, A.; Nui, Y.; Cherlet, T.; Snell, L.; Watson, P.H.; Murphy, L.C. Activated mitogen-activated protein kinase expression during human breast tumorigenesis and breast cancer progression. Clin. Cancer Res. 2002, 8, 1747-1753.

34. Davidovich, S.; Ben-Izhak, O.; Shapira, M.; Futerman, B.; Hershko, D.D. Over-expression of Skp2 is associated with resistance to preoperative doxorubicin-based chemotherapy in primary breast cancer. Breast Cancer Res. 2008, 10, R63.

35. Ravaioli, A.; Monti, F.; Regan, M.M.; Maffini, F.; Mastropasqua, M.G.; Spataro, V.; Castiglione-Gertsch, M.; Panzini, I.; Gianni, L.; Goldhirsch, A. p27 and Skp2 immunoreactivity and its clinical significance with endocrine and chemo-endocrine treatments in node-negative early breast cancer. Ann. Oncol. 2008, 19, 660-668.

36. Dai, S.; Wan, T.; Wang, B.; Zhou, X.; Xiu, F.; Chen, T.; Wu, Y.; Cao, X. More efficient induction of HLA-A*0201-restricted and carcinoembryonic antigen (CEA)-specific CTL response by immunization with Exosomes prepared from heat-stressed CEA-positive tumor cells. Clin. Cancer Res. 2005, 11, 7554-7563.

(C) 2013 by the authors; licensee MDPI, Basel, Switzerland. This article is an open access article distributed under the terms and conditions of the Creative Commons Attribution license (http://creativecommons.org/licenses/by/3.0/). 